\title{
CONCENTRAÇÃO SÉRICA DE GLICOSE, COLESTEROL, TRIGLICERÍDEOS E FRUTOSAMINA EM CADELAS GESTANTES
}

[Serum glucose, cholesterol, triglycerides and fructosamine in pregnant dogs]

\author{
Thaiza Cristina Fonseca de Figueiredo ${ }^{1}$, Daiane Poletto ${ }^{1}$, Valéria Régia Franco Sousa ${ }^{2}$, Adriane Jorge \\ Mendonça², Arleana do Bom Parto Ferreira de Almeida ${ }^{2 *}$ \\ ${ }^{1}$ Programa de Residência Uniprofissional em Medicina Veterinária - Universidade Federal de Mato Grosso \\ (UFMT). \\ ${ }^{2}$ Faculdade de Medicina Veterinária (FAVET) - UFMT.
}

\begin{abstract}
RESUMO - O objetivo desta pesquisa foi avaliar a ocorrência de diabetes mellitus em cadelas gestantes, bem como possíveis fatores associados. Foram avaliadas 20 cadelas gestantes, com idades variadas, independente de raça e período gestacional. Dosagem sérica de glicose, colesterol, triglicerídeos e frutosamina foram realizadas em pacientes em jejum de 12 horas, além de ultrassonografia abdominal. A média de glicose, colesterol e triglicerídeos obtidas foram de $87,1 \mathrm{mg} / \mathrm{dL}, 213,4 \mathrm{mg} / \mathrm{dL}$ e $132,75 \mathrm{mg} / \mathrm{dL}$, respectivamente. A concentração sérica de frutosamina foi considerada satisfatória em todas as gestantes. Apesar de não ter detectado a diabetes mellitus nas cadelas gestantes pesquisadas, a dieta e aumento de colesterol e triglicerídeos em algumas gestantes reforçam a necessidade de conscientização dos proprietários acerca dos fatores associados à esta enfermidade evitando risco as cadelas gestantes e filhotes.
\end{abstract}

Palavras-Chave: cão; gestação, glicemia; diabetes.

\begin{abstract}
The aim of this study was to evaluate the occurrence of diabetes mellitus in pregnant dogs, as well as associated factors. They evaluated 20 pregnant dogs with varying ages, regardless of race and pregnancy. Serum glucose, cholesterol, triglycerides and fructosamine were performed with the patient fasted 12 hours, and abdominal ultrasound. The mean glucose, cholesterol and triglycerides were obtained 87,1mg/dL, 213,4mg/dL and $132,75 \mathrm{mg} / \mathrm{dL}$, respectively. Serum fructosamine was considered satisfactory in all pregnant women. Despite not having detected diabetes mellitus in the surveyed bitches diet and increased cholesterol and triglycerides in some pregnant women underscore the need for awareness of the owners of the factors associated with this disease by avoiding risk pregnant women and young dogs.
\end{abstract}

Keywords: dog; pregnancy; glycemia; diabetes.

\footnotetext{
* Autor para correspondência. E-mail: arleferreira@ gmail.com Recebido: 26 de fevereiro de 2016.

Aceito para publicação: 01 de abril de 2016.
} 


\section{INTRODUÇÃO}

Diabetes mellitus é um distúrbio metabólico que resulta de uma redução de níveis séricos da insulina (hipoinsulinemia) (Davison, 2015), de caráter multifatorial, tem predisposição genética, infecções, obesidade, hormônios diabetogênicos como fatores predisponentes (Farias, 2007). Em cães a ocorrência de Diabetes mellitus tipo I dependente de insulina é mais comum e a do tipo II, não dependente de insulina, é tida como rara, sendo sua maior ocorrência em gatos (Nelson \& Reusch, 2014).

Diabetes reversível ou transitório, apesar de incomum, é descrito em cães, sendo as fêmeas mais predispostas devido à maior exposição a hormônios diabetogênicos como progesterona, hormônio do crescimento, lactogênio placentário e citocinas placentárias, que reduzem a sensibilidade de órgãos alvos à ação da insulina (Catchpole et al., 2008 Jericó et al., 2014). Neste contexto, a diabetes mellitus gestacional apresenta relevante importância (Catchpole et al., 2008) e é similar a doença humana, está associada à idade avançada, histórico familiar e excesso de peso (Phillips \& Jeffries, 2006).

De acordo com Johnson (2008) a gestação afeta os mecanismos de regulação da glicose de quatro formas: 1. promovendo resistência à insulina, 2 . suprimindo o transporte intracelular de glicose, 3 . levando a redução na sua utilização, além disso, 4. ocorre uma deficiência de energia intracelular relativa aumentando a concentração sérica de glicose. Quando esses mecanismos ocorrem de forma intensa pode resultar em Diabetes mellitus gestacional que, em alguns casos, a sensibilidade à insulina pode chegar à $43 \%$. O perfil hormonal durante a gestação em cadelas está associado ao aumento de progesterona no plasma de $1 \mathrm{ng} / \mathrm{ml}$ (anestro) para níveis superiores de $45 \mathrm{ng} / \mathrm{mL}$ durante a gestação (Pöpple et al., 2013). No terço final da gestação, os níveis séricos de prolactina (hormônio diabetogênico) estão elevados o que contribui para a resistência à insulina (Connolly et al., 2004).

Para o diagnóstico desta afecção a presença de sinais clínicos condizentes, hiperglicemia persistente em jejum e glicosúria firmam a suspeita, além da mensuração de frutosamina sérica (Jericó et al., 2014). Achados laboratoriais como aumento das concentrações séricas de alanina aminotransferase (ALT) e fosfatase alcalina (FA), assim como: aumento nas concentrações plasmáticas de triglicérides, colesterol, lipoproteínas, quilomicrons e ácidos graxos livres, podem ser observados e na ultrassonografia abdominal é possível detectar a presença de macrossomia fetal, fator complicador da Diabetes mellitus gestacional (Jacob et al., 2014).

Diante disto, o presente trabalho tem como objetivo investigar os níveis séricos de glicose, colesterol, triglicerídeos e frutosamina em cadelas gestantes atendidas em Hospital Veterinário da Universidade Federal de Mato Grosso.

\section{MATERIAIS DE MÉTODOS}

Foram selecionadas cadelas gestantes, independente da idade, raça e período gestacional atendidas em Hospital Veterinário da Universidade Federal de Mato Grosso, campus Cuiabá. Durante a consulta foi realizado exame clínico geral e informações de possíveis fatores associados à diabetes gestacional como predisposição genética, alimentação, obesidade, uso anterior de anticoncepcional, e doenças concomitantes à gestação foram questionados e anotados em uma ficha, após prévia autorização do proprietário.

Após jejum de 12 horas, foi realizada a coleta de sangue, cerca de $5 \mathrm{ml}$ com anticoagulante para obtenção de soro para dosagem sérica de frutosamina pelo analisador bioquímico semiautomático (SB 190 CELM®) com kit comercial. A dosagem de glicose, colesterol e triglicerídeos foi realizada com o aparelho portátil Accutrend $\mathrm{R}$ Plus ${ }^{\circledR}$, segundo recomendações do fabricante. Os valores de referências utilizados foram os descritos por Kaneko et al. (1997). Na ocorrência de hiperglicemia foram coletadas amostras de urina por sondagem uretral ou cistocentese, esta última de forma asséptica, e processadas segundo Kantek \& Navarro (1996) para urinálise (avaliação físicoquímica e de sedimento) com uso do Combur ${ }^{{ }^{\circ}}$ Test M.

Para avaliação do período gestacional, viabilidade e macrossomia fetal foi realizado ultrassonografia abdominal utilizando o aparelho Esaote modelo Mylab 30.

Os dados obtidos foram tabulados para análise descritiva e análise das médias, pelo programa Excel, dos testes laboratoriais empregados na pesquisa.

\section{RESULTADOS E DISCUSSÃO}

Nesta pesquisa foram avaliadas 20 cadelas gestantes. Destas, $6(30 \%)$ eram sem raça definida e $14(70 \%)$ com raça definida, sendo elas Shit-zu (4), Pinscher (4), Labrador (1), Pug (1), Boxer (1), Beagle (1), Maltês (1) e Spitz Alemão (1). Fall et al. (2010) descrevem as raças Yorkshire Terrier, Labrador Terrier, Husky Siberiano, Elkhounds Sueco e Norueguês, Malamute do Alaska, Border 
Collie sendo acometidas por diabetes mellitus gestacional. Destas já descritas, apenas Labrador Terrier foi avaliado nesta pesquisa. Estudos descrevem a predisposição de algumas raças ao desenvolvimento de diabetes e uma maior ocorrência em fêmeas comumente associado ao diestro e gestação (Catchpole et al., 2013).

Segundo Fall et al. (2010) e Mared et al. (2012) as cadelas idosas tendem a ser mais frequentemente diagnosticadas com diabetes durante o diestro e gestação quando as concentrações de progesterona e hormônio do crescimento estão aumentadas, no entanto, isto não foi observado na presente pesquisa onde todas as cadelas gestantes, independente da idade, não apresentaram diabetes.
A média da glicemia sérica das cadelas gestantes pesquisadas foi de $87,1 \mathrm{mg} / \mathrm{dL}$, com valor mínimo e máximo de $53 \mathrm{mg} / \mathrm{dL}$ e $123 \mathrm{mg} / \mathrm{dL}$, respectivamente. Segundo (Pöpple et al., 2013) o aumento nos níveis de progesterona, superiores a $45 \mathrm{ng} / \mathrm{mL}$ na gestação pode induzir a uma baixa sensibilidade dos tecidos a insulina levando consequentemente à hiperglicemia. Apesar desta afirmação, tal característica não foi observada nesta pesquisa, pois $75 \%$ das cadelas apresentavam-se normoglicêmicas e apenas uma foi encontrada com hiperglicemia (Tabela 1). Aliado a isso no terço final da gestação, os níveis séricos do hormônio diabetogênico prolactina estão elevados o que contribui para a resistência à insulina (Connolly et al., 2004). Das gestantes inseridas no projeto apenas $4(20 \%)$ delas encontravam-se no terço final da gestação, não sendo observado hiperglicemia em nenhuma delas.

Tabela 1. Classificação das cadelas gestantes de acordo com a dosagem de glicemia realizada com 12 horas em jejum.

\begin{tabular}{lcc}
\hline & Número cadelas gestantes & $\%$ de cadelas gestantes \\
\hline Hipoglicemia & 04 & $20 \%$ \\
Normoglicemia & 15 & $75 \%$ \\
Hiperglicemia & 01 & $5 \%$ \\
\hline
\end{tabular}

Valores: Hipoglicemia (<70mg/dL); Normoglicemia (70-110mg/dL); Hiperglicemia (> 110mg/dL).

O aumento de glicose sanguínea em casos de estresse, agitação, excitabilidade pode levar ao falso diagnóstico de diabetes mellitus, desta forma a dosagem de frutosamina sérica possibilita monitorar os níveis de glicose das últimas três semanas (Jericó et al., 2014). O encontro de 100\% das cadelas gestantes com níveis satisfatórios de frutosamina (Tabela 2) reforça os níveis de glicose também normais nas mesmas, inclusive na gestante hiperglicêmica inferindo possível aumento transitório da glicose sanguínea. A presença de glicosúria que poderia confirmar a diabetes (Nelson \& Reusch, 2014) não pode ser realizada por não autorização do proprietário.

Tabela 2. Níveis de frutosamina sérica nas cadelas gestantes pesquisadas.

\begin{tabular}{cccc}
\hline Níveis de frutosamina & Valores $(\mathrm{mmol} / \mathrm{L})$ & Cadelas gestantes $(\mathrm{n})$ & Cadelas gestantes $(\%)$ \\
\hline Satisfatório & $\leq 3,2 \mathrm{mmol} / \mathrm{L}$ & 20 & $100 \%$ \\
Medíocre & $\geq 3,2$ a $3,7 \mathrm{mmol} / \mathrm{L}$ & & \\
Insatisfatório & $>3,7 \mathrm{mmol} / \mathrm{L}$ & & \\
\hline
\end{tabular}

Similar à doença humana, fatores como obesidade e alimentação podem predispor à resistência a insulina e ocorrência de diabetes mellitus (Fall et al., 2008; Fall et al., 2010). Das gestantes pesquisadas, nove alimentavam-se apenas de ração, outras nove, associação de comida caseira a ração e duas, a alimentação era exclusivamente comida caseira. $\mathrm{O}$ aumento de progesterona e hormônio do crescimento leva a resistência a insulina e a carboidratos (Fall et al., 2008; Fall et al., 2010), sendo a dieta rica em carboidratos complexos e fibras, importante no período gestacional visando minimizar o risco de diabetes. Nenhuma das cadelas gestantes apresentava-se obesa no momento da pesquisa, fator de risco para o diabetes (Faria, 2007; Nelson \& Reusch, 2014).

No entanto, a média de colesterol das cadelas pesquisadas foi de $213,4 \mathrm{mg} / \mathrm{dL}$, com mínima de
$120 \mathrm{mg} / \mathrm{dL}$ e máxima de $286 \mathrm{mg} / \mathrm{dL}$ e a média de triglicerídeos foi de $132,75 \mathrm{mg} / \mathrm{dL}$, com mínima < 70 e máxima > 600mg/dL. Com base nas referências de Kaneko et al. (1997) a média encontrada de colesterol e triglicerídeos encontra-se elevado para triglicerídeos, sendo $25 \%$ e $30 \%$ das cadelas apresentando aumento de colesterol e triglicerídeos, fator preocupante tendo em vista as complicações associadas a elevação destes no organismo, sendo uma delas a diabetes (German, 2006).

\section{CONCLUSÃO}

Apesar de não ter detectado a diabetes mellitus nas cadelas gestantes pesquisadas, a dieta e aumento de colesterol e triglicerídeos em algumas gestantes reforçam a necessidade de conscientização dos proprietários acerca dos fatores associados à esta 
enfermidade evitando risco as cadelas gestantes e filhotes.

\section{COMITÊ DE ÉTICA}

Este trabalho foi submetido e aprovado pela Comissão de Ética de Uso de Animais CEUA/UFMT protocolo de número 23108.075942/2015-33.

\section{REFERÊNCIAS}

CATCHPOLE, B. et al. Canine diabetes mellitus: from phenotype to genotype. Journal of Small Animal Practice, v. 49, n. 1, p. 4-10, 2008.

CATCHPOLE, B. et al. Genetics of canine diabetes mellitus: Are the diabetes susceptibility genes identified in humans involved in breed susceptibility to diabetes mellitus in dogs? The Veterinary Journal, v. 195, n. 2, p. 139-147, 2013.

CONNOLLY, C. C. et al. Insulin action during late pregnancy in the conscious dog. American Journal of Physiology: Endocrinology and Metabolism, v. 286, n. 6, p. E909-15, 2004.

DAVISON, L. J. Diabetes mellitus and pancreatitis - cause or effect? Journal of Small Animal Practice, v. 56, n. 1, p. 50-59, 2015 .

FALL, T. et al. Gestational diabetes mellitus in 13 dogs. Journal of Veterinary Internal Medicine, v. 22, p. 1296-1300, 2008.

FALL, T. et al. Diabetes Mellitus in Elkhounds Is Associated with Diestrus and Pregnancy. Journal of Veterinary Internal Medicine, v. 24, p. 1322-1328, 2010.

FARIA, P. F. Diabetes Mellitus em Cães. Acta Veterinaria Brasílica, v. 1, n.1, p. 8-22, 2007.

GERMAN, A. J. The Growing Problem of Obesity in Dogs and Cats. The Journal of Nutrition, v. 136, p.1940S-1946S, 2006.

JACOB, T. A. et al. Diabetes mellitus gestacional: uma revisão de literatura. Brazilian Journal of Surgery and Clinical Research, v. 6, n. 2, p. 33-37, 2014.

KANEKO, J. J. Clinical biochemistry of domestic animals. $5^{\text {th }}$ edition. Gulf Professional Publishing. 932p, 1997.

JERICÓ, M. M. et al. Tratado de Medicina Interna de Cães e Gatos. São Paulo: Roca, p. 1747-1752, 2014

JOHNSON, C. A. Glucose homeostasis during canine pregnancy: Insulin resistance, ketosis, and hypoglycemia. Science Direct Theriogenology, v. 70, p. 1418-1423, 2008.

KANTEK, G.; Navarro, C. E. Manual de urinálise veterinária São Paulo: Varela, 96p, 1996.

MARED, M. Evaluation of circulating concentrations of glucose homeostasis biomarkers, progesterone, and growth hormone in healthy Elkhounds during anestrus and diestrus. American Journal of Veterinary Research, v. 73, p. 242-247, 2012.

NELSON R.W.; REUSCH C.E. Animal models of disease: Classification and etiology of diabetes in dogs and cats. Journal of Endocrinology, v. 222, p. T1-T9, 2014.

PHILLIPS, P. J.; JEFFRIES, B. Gestational diabetes. Worth finding and actively treating. Australian Family Physician, v. 35 , p.701-703, 2006.
PÖPPLE, A.G., et al. Diabetes Mellitus remisson after resolution of inflammatory and progesterone - related conditions in bitches. Research in Veterinary Science, v. 94, p. 471-473, 2013. 